\title{
A New Conformal FDTD for lossy thin panels
}

\author{
Miguel R. Cabello, Luis D. Angulo, J. Alvarez, Member, IEEE, A. Rubio Bretones, \\ and Salvador G. Garcia, Senior Member, IEEE
}

\begin{abstract}
A novel conformal technique for the finite difference time domain (FDTD) method is proposed to deal with curved and arbitrarily oriented lossy thin panels. This is formulated as an extension of the existing conformal relaxed Dey-Mittra method (CRDM) combined with sub-cell algorithms to handle wave propagation across the panel. Two alternatives are presented for this: the classical impedance network boundary condition (INBC), and a recent sub-gridding boundary condition (SGBC) developed by the authors. Several test cases are employed to demonstrate the capability of the proposed method to remove errors associated with the usual staircased FDTD method.
\end{abstract}

Index Terms-Conformal modeling, shielding effectiveness (SE), electromagnetic shielding, finite-difference time domain (FDTD), lossy materials, sub-cell models, thin panels

\section{INTRODUCTION}

Since its original formulation in 1966 [1], the finite difference time domain (FDTD) method remains the most widely used numerical method to solve Maxwell's curl equations in time domain. We can attribute this success, at least in part, to two strong points: first, its computational efficiency and, second, the simplicity of its implementation.

Despite the many advantages of the structured distribution of the FDTD fields, it also poses serious constraints when we seek to resolve multi-scale problems. For instance, when small thin structures are involved, the classical FDTD requires the use of small space steps and this, in turn demands greater computer memory and CPU time. Even if mesh-size reductions enhance the accuracy in general, staircasing presents inherent errors associated to the enlargement of the electrical size of the simulated objects, shifting their resonance frequencies, and adding extra ohmic losses for conductive objects, which, in practice, degrades the FDTD second order of convergence to quasi-first order [2].

A typical workaround to avoid brute-force meshing around curved regions is the use of local subcell methods. Two main approaches can be found in the literature: subgridding techniques [3]-[5], and Conformal Dey-Mittra (DM) methods [6]. Subgridding techniques employ a refined mesh in the neighborhood of the curvature which is connected to usual coarse mesh using interpolation techniques at their boundary. In some schemes, the refined mesh can be evolved with a local time-stepping algorithm, driving to a great increase in computational efficiency [5]. They are very helpful to handle

The 1st 2nd, 4th and 45th authors are with the Dept. of Electromagnetism, University of Granada, Fuentenueva s/n, 18071 Granada, Spain. The 3rd author is with AIRBUS DS, Avd. John Lennon s/n. Getafe (Spain). Corresponding author e-mail: salva@ugr.es

The work described in this paper and the research leading to these results was supported by the Spanish MINECO, EU FEDER under Project TEC201679214-C3-3-R (MINECO, Spain), P12-TIC-1442 (Junta de Andalucia, Spain), and Alhambra-UGRFDTD (AIRBUS). intricate intra-cell details and can be applied recursively. However, as will be shown in this paper, they do not remove some of the fundamental errors which are inherent to staircasing. On the contrary, DM conformal methods allow cells to be traversed by curved surfaces which split the cell into two regions. The technique involves modifying the coefficients on the original FDTD, weighting the corresponding fields with the areas of their respective regions. A drawback of the original DM method is its more restrictive stability condition, since the maximum time step for its stability is bounded by the minimum size of the globally split conformal regions. To address these issues, a variant of the DM method, the conformal relaxed Dey-Mittra (CRDM) method was proposed in [7] for perfect electrically conducting (PEC) surfaces. This method describes how to build a conformal mesh by taking control over the trade-off between the accurate representation of the original geometry and a maximum time-step chosen for computer affordability. Notably, this method retained a quasi-second order of convergence for the analyzed cases. In summary, DM methods excel when dealing with smooth intra-cell curvatures by providing a better approximation to the curved portion of the object inside a Yee cell, naturally overcoming the staircasing errors.

Materials consisting of lossy thin panels are another case requiring a subcell approach in FDTD. A typical method to deal with these materials consists of a modification of the classical surface impedance boundary conditions, to take into account both scattering and penetration by means of two or four-port impedance network boundary conditions (INBCs) [8], [9]. An alternative based on the so-called subgriding boundary conditions (SGBCs) was presented in [10], [11], as a mean to overcome stability issues found in INBC.

In these previous works, the panels had to be aligned with the Cartesian mesh, in this work, we present a novel technique which extends the CRDM method to deal with curved lossy thin panels, using either SGBC or INBC to model its internal behavior. The proposed method is first validated with two sets of test cases: a low-frequency (LF) conduction problem by lossy strips slanted at different angles with the Cartesian mesh, for which the Direct Current (DC) resistance is found; and a broadband shielding problem by a spherical enclosure, for which the shielding effectiveness is evaluated. A last complex test case consisting on the calculation of the currents coupled to a cable shield inside an aircraft, serves to confirm the accuracy and to test the robustness of the method.

The rest of this paper is organized as follows: In Section II, the proposed method is traced; In Section III, the fundamentals of the INBC and SGBC methods are recalled. In Section $\mathrm{V}$, results for the two aforementioned test cases are shown. Finally, Section VI presents the conclusions. 


\section{CRDM FOR THIN PANELS}

The approach combining subcell methods to treat curved lossy thin panels with the conformal method [10] can be described in a unified manner regardless of the treatment for the thin pannel, either SGBC or INBC, which only differ in the way they relate the tangential fields on each side of the slab. The overall scheme is a generalization of the conformal technique used in [7] for PEC. Now, we need to consider two tangential E-field components on each side of the panel instead of taking it to be null as for PEC. Therefore, two new degrees of freedom, $E_{S, 1}$ and $E_{S, 2}$ are introduced, apart from the usual DM H-field $H_{S, 1}$ and $H_{S, 2}$ components at the centroid of each sub-cell split by the boundary. A local time advance algorithm is employed in conformal cells (with the same global time step), which can be summarized as follows:

1) The E-fields $E_{k, \nu}^{n}$ at a given time step $n$, along the edge $\nu$ of the cell on side $k$ with respect to the thin panel, are updated as usual; according to the usual DM algorithm [6] by finite differences involving the adjacent magnetic fields.

2) The E-fields $E_{S, 1}, E_{S, 2}$ along both sides of the material boundary are updated by the thin-panel relationships by using either INBC and SGBC, as described in Sections III-A and III-B.

3) The H-fields $H_{S, 1}$ and $H_{S, 2}$ located at the centroid of each sub-cell split by the boundary are updated using the closed-line integral of tangential electric fields along their contour,

$$
\begin{aligned}
& H_{S, 1}^{n+1 / 2}=H_{S, 1}^{n-1 / 2}-\frac{\Delta t}{\mu_{0} A_{1}}\left(\Gamma_{1}^{n}+l_{S} E_{S, 1}^{n}\right) \\
& H_{S, 2}^{n+1 / 2}=H_{S, 2}^{n-1 / 2}-\frac{\Delta t}{\mu_{0} A_{2}}\left(\Gamma_{2}^{n}-l_{S} E_{S, 2}^{n}\right)
\end{aligned}
$$

where $\Gamma_{k}^{n}=\sum_{\nu} l_{k, \nu} E_{k, \nu}^{n}$ is the usual discrete line integral of the E-field components for conformal edges where $l_{S}$ is the conformal edge length.

The rest of the advancing algorithm for cells not affected by material traversing are found by the Yee-FDTD method, as usual.

\section{THIN PANEL MODELING}

As stated, the two tangential E-field components along the thin panel are introduced to be calculated either by SGBC or INBC. The difference between these methods depend on the wave propagator used. INBC finds the E-fields on the surface by a two-port, four-port if anisotropic, network relationship between the adjacent $\mathrm{H}$-fields [9], [12], whereas SGBC expressly propagate in time the EM fields inside the slab. Both methods rely on the fact that waves propagate along the direction perpendicular to the slab as a TEM mode, regardless of the incidence angle of waves impinging on it. This assumption holds as far as the refractive index inside the slab is much higher than outside, which is the case for lossy panels (see [10] for a further discussion). Let us briefly recall the fundamentals of each technique, assuming for simplicity isotropic media.

\section{A. Impedance Network Boundary Conditions}

INBC relates the tangential $\mathrm{E}$ and $\mathrm{H}$-field components by frequency-domain $\mathrm{Z}$ parameters. These can be found analytically either from the S-parameters or from the constitutive relationships of the panels [13], [14]. Fig. 1 shows the relationship for an isotropic thin-panel in the conformal case. The adjacent $\mathrm{H}$-field components are assumed to be found using (1), and the electric fields are found as a function of them as

$$
\left[\begin{array}{l}
E_{S, 1} \\
E_{S, 2}
\end{array}\right]=\left[\begin{array}{ll}
Z_{11} & Z_{12} \\
Z_{21} & Z_{22}
\end{array}\right]\left[\begin{array}{c}
H_{S, 1} \\
-H_{S, 2}
\end{array}\right]
$$

where $E_{S, 1}$ and $H_{S, 1}$ are the fields on one side of the thin panel and $E_{S, 2}$ and $H_{S, 2}$ are the fields on the other. To implement the frequency-domain relationship (2) into time domain, a typical approach is first to expand each matrix element $Z_{i j}$ into a sum of poles/residues partial fractions, found by a vector-fitting (VF) procedure [15], and next translate each term of the expansion into the time domain by a piecewise linear recursive convolution (PLRC) algorithm [16]. It bears noting

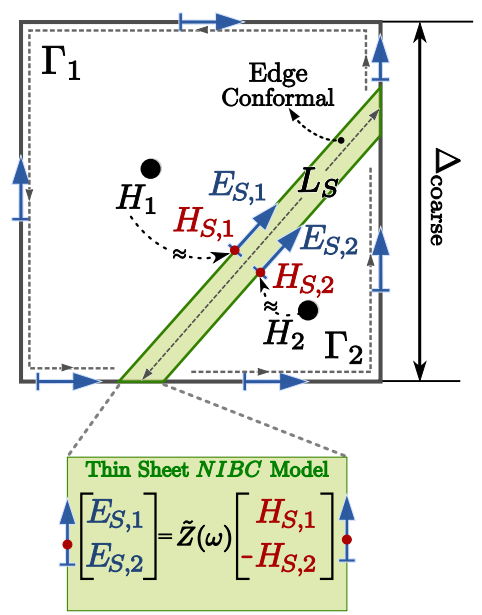

Fig. 1: Cross section of a FDTDcell with a INBC boundary.

that the INBC method presents some problems when coupled with the usual scheme of Yee-FDTD fields as it requires us to know simultaneously both the $E$ and $H$-fields at the same time and location on the interface. However, the Yee-FDTD method does not present the electric and magnetic fields co-located but staggered by semi-integer time and space increments. Thus, some kind of interpolation is needed in order to find $H_{S, 1}^{n+1}$ and $H_{S, 2}^{n+1}$, from $H_{1}^{n+1 / 2}$ and $H_{2}^{n+1 / 2}$. A typical approach [8], [9], [17] employs

$$
\begin{aligned}
& H_{S, 1}^{n+1} \simeq H_{1}^{n+1 / 2} \\
& H_{S, 2}^{n+1} \simeq H_{2}^{n+1 / 2}
\end{aligned}
$$

This approximation is often considered [18], [19] to be the source of late time instabilities that show up especially in certain complex problems. This was also the reason why the authors introduced in [11] the SGBC as a late-time stable alternative, which is briefly described in the Section III-B.

\section{B. Subgridding boundary condition}

The SGBC method finds the tangential E-fields on either side of the slab by propagating the EM fields inside it 
using a 1D subgridding FDTD algorithm along the direction perpendicular to the slab. For this, a space-step fine enough to accurately sample the wave inside at the maximum frequency of interest is used inside the slab, while keeping the usual (coarse) discretization outside of it. To prevent the time constraints imposed by this reduced space-step, we used the unconditionally stable CNTD implementation given in [10] because it proved to have superior stability properties. Note that the 3D Yee-FDTD used outside is connected with the CNTD used inside the panel in a straightforward way, respecting the non-co-located space-time nature of 3D YeeFDTD. In this way, the Yee FDTD 3D fields serve as boundary conditions of 1D CNTD, and vice-versa.

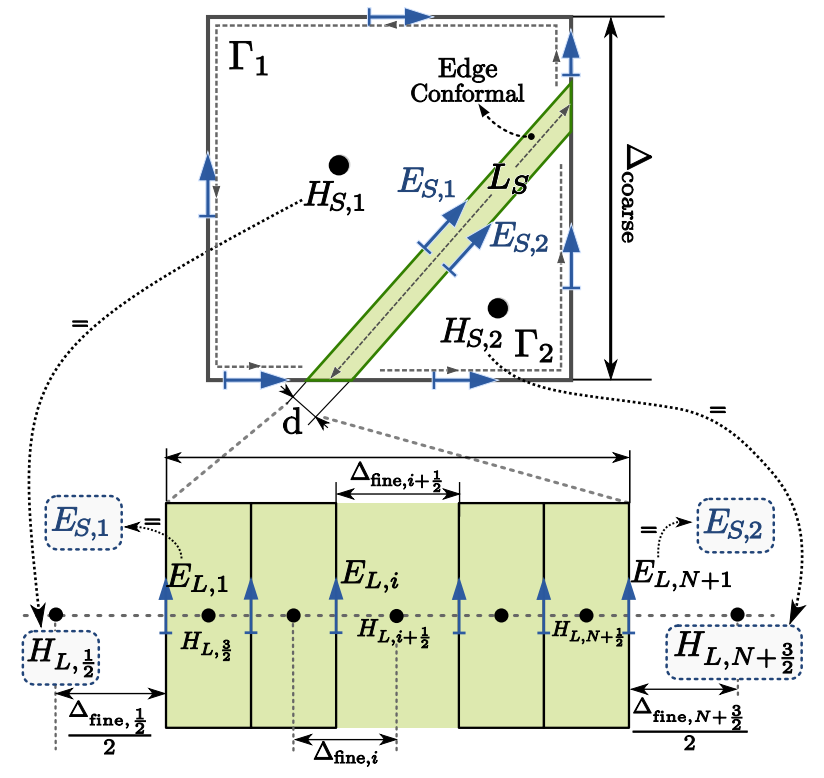

Fig. 2: Cross section of a conformal Cell with SGBC layer.

\section{Stability of SGBC}

Taking profit of the results published in [20], we can give a rough proof of the stability of the SGBC method. There, it was stated that a hybrid explicit Yee-FDTD/implicit CrankNicolson method is stable if it fulfills the Courant limit in the explicit part, and the electric tangential fields at the boundary between both regions are updated by the implicit part. The SGBC scheme described in this paper inherently fulfills these two requirements.

Actually, for a plane slab whose normal is aligned along one direction of the grid, the SGBC method is a particular and more simple case of [20]. The SGBC method is only applied in $1 \mathrm{D}$, along the direction perpendicular to the lossy slab (thanks to the transverse electromagnetic (TEM) propagation assumption [10]). Hence, the change of space steps between the two regions is a particular case of a non-uniform transition [20].

In any case, we have also verified numerically the stability of the SGBC method for a practical test case: a conductive spherical shell under plane wave incidence. The sphere has a radius of $1 \mathrm{~m}$ and a thickness of $8 \mathrm{~mm}$. It is illuminated with a uniform plane-wave with a Gaussian amplitude decaying $-3 \mathrm{~dB}$ at $1 \mathrm{GHz}$. Fig. 3 shows the ratio between the maximum time step for stability of the SGBC method $\Delta t$, and the maximum allowable one for the coarse part $\Delta t_{0}$. For conformal meshes, $\Delta t_{0}$ depends on the geometrical relaxation factor (the maximum edge-to-area ratio of the conformal cell defined in [7]). In the present case, we used a relaxation factor of 0.42. Fig. 3 shows the stability limit of the $\mathrm{CN}$ implicit unconditionally stable method. For the sake of reference, we also plot results using the usual FDTD 1D explicit conditionally stable scheme, instead of the $\mathrm{CN}$ scheme.

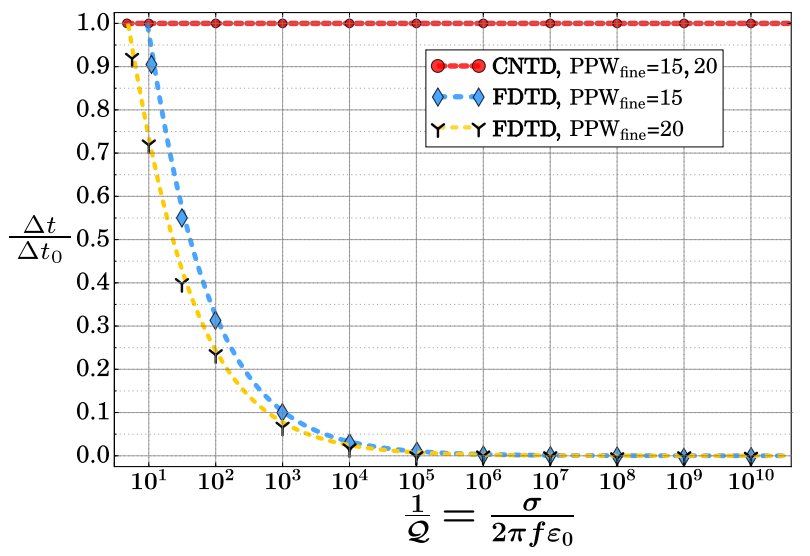

Fig. 3: Maximum time step ratio for stability: analytically (dashed line) and numerically (markers points).

To obtain the results of Fig. 3, the coarse space step is set to $\Delta_{\text {coarse }}=15 \mathrm{~mm}$, and the fine space step $\Delta_{\text {fine }}$ inside of the SGBC is chosen according to the resolution of its wavelength

$$
\Delta_{\text {fine }}=\frac{\lambda_{\text {fine }}}{\mathrm{PPW}_{\text {fine }}}
$$

where PPW denotes the number of points per wavelength at $1 \mathrm{GHz}$, and the wavelength inside the material is expressed as function of its quality factor $Q$,

$$
\lambda_{\text {fine }}=\frac{\lambda_{0}}{\sqrt{\frac{1}{2} \sqrt{1+Q^{-2}}+1}}
$$

From the results of Fig. 3, we conclude that:

1) SGBC with explicit FDTD 1D advancing scheme: The time step is restricted by the SGBC region. Actually, the numerical stability limit is in accordance with that imposed by the SGBC isolated region, $\Delta t=\Delta_{\text {fine }} / c_{0}$.

2) SGBC with implicit CNTD 1D advancing scheme: The time step is imposed by the coarse grid $\Delta t=\Delta t_{0}$, since the CNTD does not add any extra constraint.

\section{Numerical Results}

Three kinds of validations have been devised in this paper to demonstrate the accuracy of the conformal method compared to the staircased one. The first validation is based on the calculation of the quasi-static resistance of a thin strip, tilted with respect to the Cartesian axis. The error associated with the overestimation of the ohmic losses for the structured case is revealed. The second test case consists of a spherical lossy enclosure shell under plane-wave incidence, to reveal 
the underestimation of the field coupled inside (as much as $4-6 \mathrm{~dB}$ ), the shifting of their natural resonance frequencies with respect to the physical ones, and the appearance of numerical artifacts in the form of spikes due to the staircased discretization. The third validation presents a more challenging and complex test case used to assess high intensity radiated field (HIRF) effects on a cable shield running inside an aircraft.

The three test cases presented herein have been simulated using SGBC and INBC, with a similar accuracy (no instabilities have been found for the INBC in these particular cases.

\section{A. Resistance of thin strips}

The prediction of the DC resistance of a slanted strip serves well to illustrate the source of errors introduced by the staircased mesh used by classical FDTD. The DC resistance of a conductive strip is well known to be

$$
R_{\mathrm{DC}, \text { teo }}=\frac{1}{\sigma} \frac{L_{S}}{w \mathrm{~d}}
$$

where $L_{S}$, is its length, $w$ is the width and $\mathrm{d}$ is the thickness of the conductor strip. However, $L_{S}$ depends on how the strip is meshed, which can be easily understood by realizing that the staircased rectilinear grid imposes a Manhattan distancebased metric, also defined as a $1^{\text {th }}$ order Minkowski distance. For instance, if $\vec{p}_{1}$ and $\vec{p}_{2}$ are two points unaligned with a Cartesian axis, with coordinates in $2 \mathrm{D}\left(x_{1}, y_{1}\right)$ and $\left(x_{2}, y_{2}\right)$, the Manhattan distance is $\left|x_{1}-x_{2}\right|+\left|y_{1}-y_{2}\right|$, always larger than the Euclidean one. In general, the associated relative error is

$$
\text { err }=\frac{\left|d_{\text {manhattan }}-d_{\text {euclidean }}\right|}{d_{\text {euclidean }}}=\frac{\sum_{k}^{N_{D}}\left|p_{1, k}-p_{2, k}\right|}{\sqrt{\sum_{k}^{N_{D}}\left(p_{1, k}-p_{2, k}\right)^{2}}}-1
$$

with $N_{D}$ being the spatial dimension. It bears noting that this error is independent of the grid step size, so that staircased refinements, or even Cartesian subgridding, are not able to mitigate it (Fig. 4). The relative error only depends on the alignment of the points $p_{1,2}$ with respect to the grid with a maximum value for diagonal-wise structures given by

$$
\mathrm{err}=\frac{N_{D}}{\sqrt{N_{D}}}-1
$$

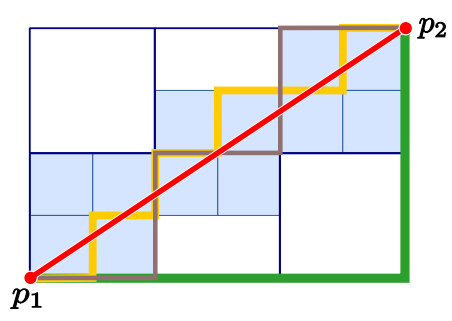

Fig. 4: The red line represents the diagonal Euclidean distance between two points $p 1, p 2$. Green, brown, and yellow lines have the same Manhattan distance even for different grid sizes.

To study this effect, we have used the numerical test setup illustrated in Fig. 5 consisting of a conductive strip, connected at each end to an external U-shaped set of PEC strips, in turn excited by a hard voltage source uniformly distributed inside a delta-gap.

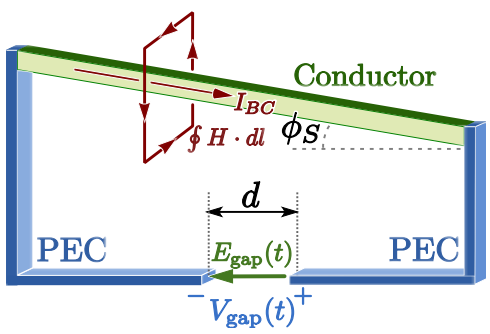

Fig. 5: Test setup for estimating the DC resistance.

The used voltage source has a quasi-DC time profile: it starts at $t=0$ with a Gaussian profile $e^{-\left(t-t_{0}\right)^{2} /\left(2 s^{2}\right)}$, with $s=0.027 \mu \mathrm{s}$, achieving a maximum value of $1 \mathrm{~V}$ at $t_{0}=$ $0.1 \mu s$, after which it is kept constant in time. The resistance is computed by dividing the value of the voltage drop across the gap, by the bulk current $I_{B C}$ computed by integrating the H-fields along a loop fully enclosing the stripe (in red in Fig. 5): $I_{\mathrm{BC}}=\oint H \cdot d l$. The whole setup can be enclosed, with no additional discretization error, inside a PEC cage in order to speed up the calculations, since no error of reflections are introduced by these at the DC limit.

$$
R_{\mathrm{DC}, \mathrm{sim}}=\frac{V_{\text {gap }}}{I_{\mathrm{BC}}}=\frac{E_{\text {gap }} d_{\text {gap }}}{\oint H \cdot d l}
$$

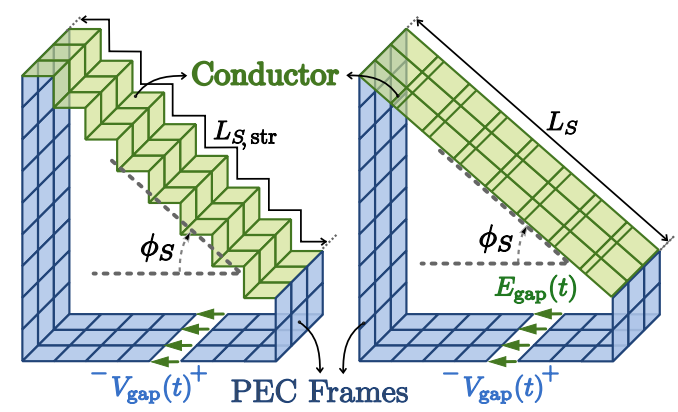

Fig. 6: Discrete test-setup for the computation of the DC resistance of a conductive thin strip slanted at angle $\phi_{S}$ with respect to the grid: staircased and conformal meshings.

The relative error of the resistance as a function of the tilting angle with respect to the Cartesian axes is illustrated in Fig. 7 both for conformal and for structured meshes. We have taken a thin strip with a conductivity of $\sigma=20 \mathrm{~S} / \mathrm{m}$, a length of $L_{S}=\left(216 \mathrm{~mm} / \cos \phi_{S}\right)$, a width of $w=120 \mathrm{~mm}$, and a thickness of $\mathrm{d}=2 \mathrm{~mm}$. We note that the error is negligible for the conformal mesh, while for the structured mesh behaves, as expected, with a theoretical value estimated by (4) employing the Manhattan distance (5) to find $L_{S}$

$$
L_{S, \mathrm{str}}=L_{S}\left(\sin \phi_{s}+\cos \phi_{s}\right)
$$

to yield,

$$
R_{\mathrm{DC}, \mathrm{str}}=\frac{1}{\sigma} \frac{L_{S}\left(\sin \phi_{s}+\cos \phi_{s}\right)}{W \mathrm{~d}}
$$


We conclude that the staircased mesh irremediably leads to errors in the resistance of unaligned objects, while conformal methods can properly account for them.

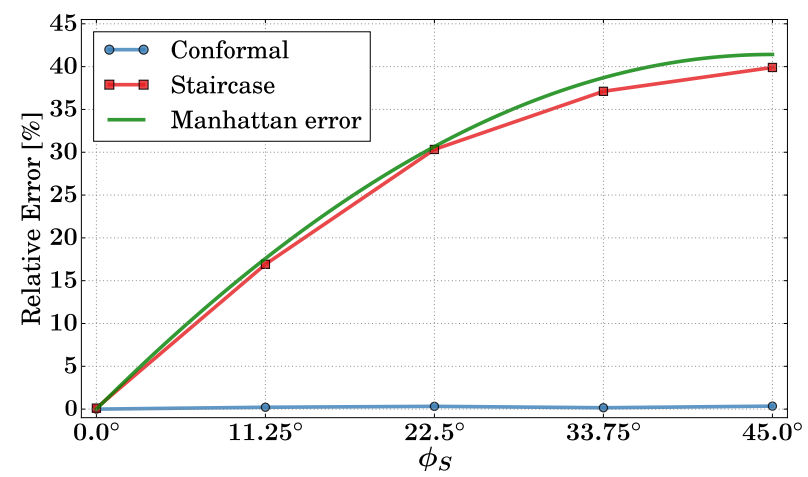

Fig. 7: Error for the estimation of the DC resistance as function of the slanting angle with respect to the grid. The Manhattan error is assumed to be the relative difference between Manhattan and Euclidean distances, given by err $=\cos \phi_{s}+\sin \phi_{s}-1$.

\section{B. Shielding effectiveness of a lossy spherical shell}

Next, the broadband penetration of the field inside a conductive spherical shell is analyzed. For this, a sphere $1 \mathrm{~m}$ in radius, with a thickness of $5 \mathrm{~mm}$, and a conductivity of $200 \mathrm{~S} / \mathrm{m}$, has been taken. The value of shielding effectiveness (SE) at the center of the sphere is evaluated, under the incidence of a uniform plane-wave with a Gaussian profile contained in a domain truncated by perfectly matched layers (PML),

$$
\mathrm{SE}_{\mathrm{dB}}=20 \log \frac{\sqrt{\vec{E}_{\text {center }} \cdot \vec{E}_{\text {center }}^{*}}}{\sqrt{\vec{E}_{\mathrm{inc}} \cdot \vec{E}_{\mathrm{inc}}^{*}}}
$$

where $\left({ }^{*}\right)$ denotes the complex conjugate, $(\cdot)$ the usual dot product, $E_{\text {inc }}$ the E-field of the incident plane-wave, and $\vec{E}_{\text {center }}$ the E-field coupled at the center of the sphere.

Fig. 8 compares the results for the standard staircase and FDTD and for the conformal technique with no apparent differences between INBC and SGBC, as found for the previous test case. Two different space increments for the space step are used for the usual FDTD method: $\Delta=5 \mathrm{~mm}, 25 \mathrm{~mm}$. Analytical predictions from [21], [22] are used for reference. We also include results found with the average method proposed in [23] extended for conductive materials. This algorithm employs the usual Yee scheme for cells partially filled with a material, but with a conductivity weighted by their void-to-filled ratio. The time-step is evaluated according to a CFLN $=0.7$, using the relaxed technique proposed in [7].

Fig. 8 shows excellent agreement between INBC and SGBC, and a mismatch for the staircased FDTD. A constant offset level in the staircased results of $5-6 \mathrm{~dB}$ can be discerned, while for the conformal approach, the offset level is lessened by as much as $1 \mathrm{~dB}$. Also the average method is accurate at low frequencies, while it fails at high frequencies. The reason for this is that it does not catch the space field variation inside the conductor at high frequencies when the skin-depth effect begins to dominate (smaller cell sizes would be necessary to account for this).

It also bears noting that spurious spikes appear at some frequencies for FDTD. These have been demonstrated [24] to be inherent to the staircased approximation of the curvature of the sphere, while they are greatly lessened by the proposed conformal method. Finally, in Fig. 9 we also show in detail the position of the first resonance using $\Delta=100 \mathrm{~mm}$, which is displaced for the staircase with respect to the analytical and conformal ones, as expected, due to the error in the actual electrical size inherent to the staircased mesh.

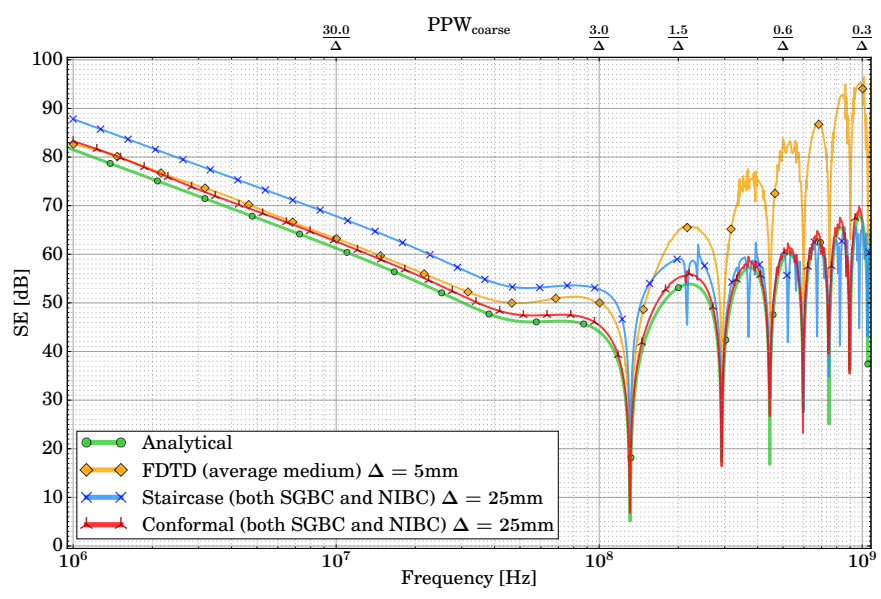

Fig. 8: SE for a sphere $1 \mathrm{~m}$ in radius and $5 \mathrm{~mm}$ thick, with $200 \mathrm{~S} / \mathrm{m}$ conductivity.

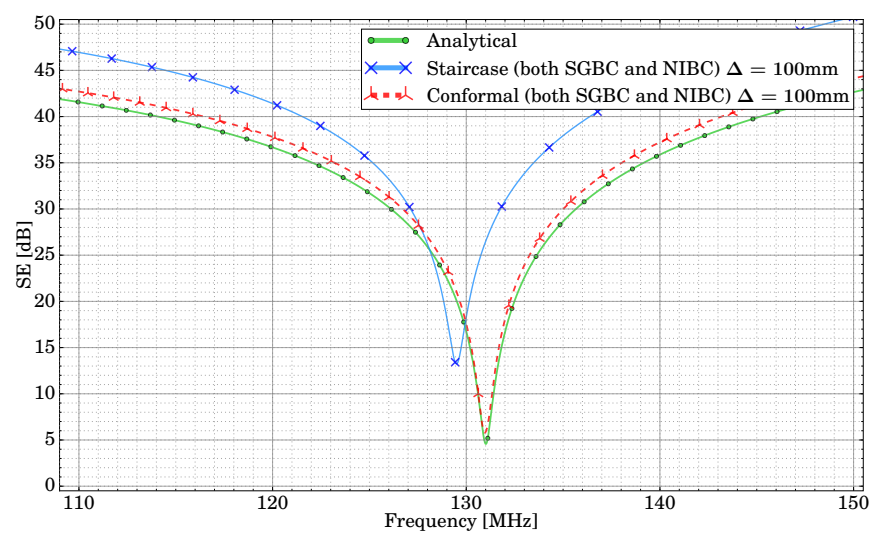

Fig. 9: SE for a sphere $1 \mathrm{~m}$ in radius and $5 \mathrm{~mm}$ thick, with $200 \mathrm{~S} / \mathrm{m}$ conductivity (detail of the first resonance).

\section{Assessment of the HIRF effects on an aircraft}

In this test case, the HIRF effects on a cable enclosure inside an aircraft are studied in a modified version of an EV55 aircraft ${ }^{1}$. The aircraft is formed by two parts: the airframe and a cable-enclosure. The airframe is assumed to be PEC, and the shield of the cable-enclosure has been modeled with a

1 The EV55-aircraft geometry presented herein has been provided by EVEKTOR and is publicly available, as a result of the FP7 HIRF-SE project, after EVEKTOR's clearance upon request to salva@ugr.es. 


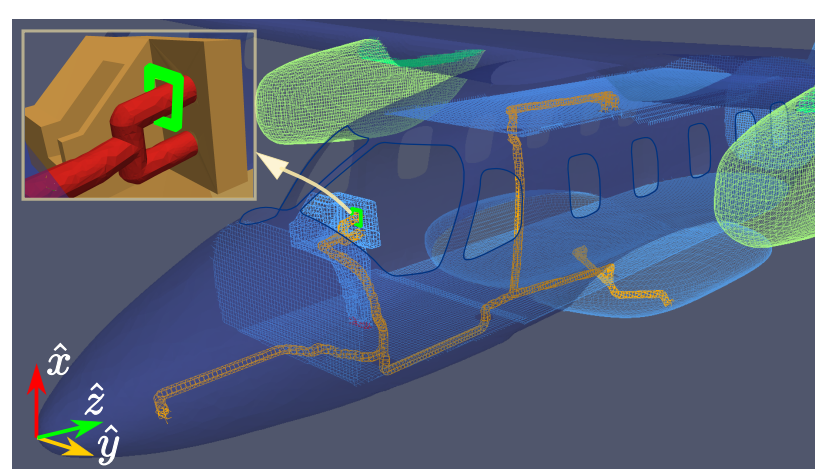

Fig. 10: Test setup for estimating the transfer function on the cable enclosure.

cylindrical shell with a mean radius of $30 \mathrm{~mm}$, a thickness of $2 \mathrm{~mm}$ and a resistance per unit length (p.u.1.) of $1.263 \mathrm{~m} \Omega / \mathrm{m}$. The thin panel methods proposed in this manuscript have been employed to deal with the coupling of EM environment inside the lossy shell of the cable-enclosure. The aircraft is illuminated in free space with a plane wave with a Gaussian amplitude decaying $-3 \mathrm{~dB}$ at $1 \mathrm{GHz}$, and an incidence of $\frac{1}{\sqrt{2}}(\hat{x}+\hat{z})$ and electric field polarization of $\frac{1}{\sqrt{2}}(\hat{x}-\hat{z})$. The aim of this test case is evaluate the transfer function between the induced current in the cable enclosure and the plane wave magnitude, the bulk current induced on the cable is assessed with a closed-loop line-integral on the magnetic field around the cable-enclosure.

Fig. 11 compares the results for the standard staircase and FDTD and the conformal technique, both use a grid of cubic cells with size of $12 \mathrm{~mm}$ and $24 \mathrm{~mm}$ respectively, and a timestep 0.8 times the Courant limit. The computational domain is truncated using PML of 10 layer. This test case was broadly cross-validated under the FP7 EU HIRF-SE project using several numerical techniques [25]. In this paper, we employ the discontinuous Galerkin time domain (DGTD) results of [26] as a reference, for its well known accuracy. As expected, we note that, in spite that the resolution chosen for staircased model is twice that taken for the conformal one, the latter shows a better agreement with DGTD. At low frequency, the error in the plateau region is dominated by the p.u.l. resistance of the cable enclosure, whereas at high frequencies, the position of the resonances are mandated by its electrical length.

\section{CONCLUSions}

The CDRM strategy has been extended to deal with lossy thin panels in FDTD in an accurate and computationally feasible way. Both the classical INBC and the novel SGBC can be used as sub-cell models of the thin panels, leading to similar accuracies in the results, apart from concerns regarding INBC stability, which have not appeared in the simple test cases presented here.

Three different scenarios have been chosen to assess the validity of the method: a low-frequency one to predict the DC ohmic resistance of thin strips, a broadband scenario to predict the penetration of electromagnetic fields through lossy shells, and a complex one to confirm its accuracy and assess

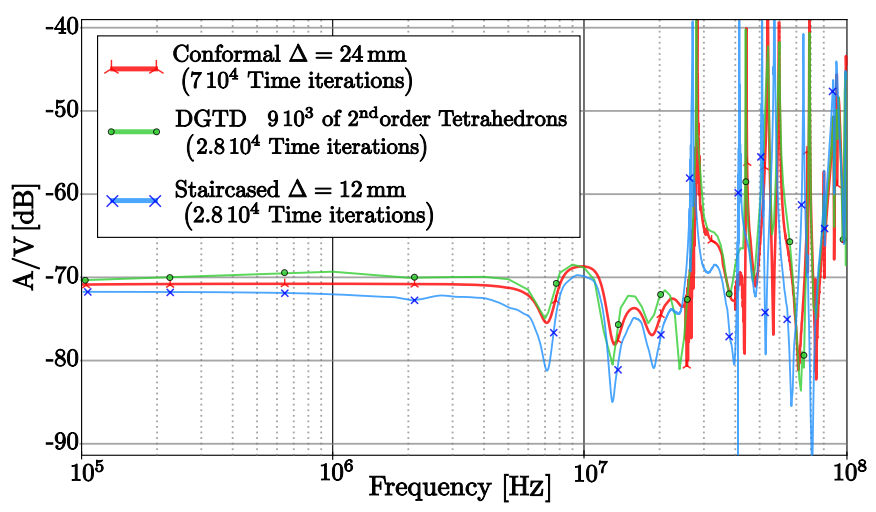

Fig. 11: Transfer functions for the current coupled to the cable enclosure of the EV55 under HIRF conditions.

its robustness. In both cases, we conclude that the conformal method overcomes the main drawback of the structured FDTD method: the overestimation of electrical sizes. This has impact on the correct estimation of ohmic losses for conduction problems, the penetration through lossy materials, and the shifting of the physical frequencies of resonance, in addition to the appearance of numerical spurious artifacts.

\section{ACKNOWLEDGMENT}

The authors wish to thank EVEKTOR for disclosing the numerical model of a modified EV55 aircraft for its simulation. They also thank J.-P. Parmantier from ONERA for his guidance in the definition, consolidation, and discussion on the EV55 numerical test case under the HIRF-SE project.

\section{REFERENCES}

[1] K. Yee, "Numerical solution of initial boundary value problems involving Maxwell's equations in isotropic media," IEEE Transactions on Antennas and Propagation, vol. 14, no. 3, pp. 302-307, May 1966.

[2] I. Zagorodnov, R. Schuhmann, and T. Weiland, "A uniformly stable conformal FDTD-method in cartesian grids," International Journal of Numerical Modelling: Electronic Networks, Devices and Fields, vol. 16, no. 2, pp. 127-141, 2003. [Online]. Available:

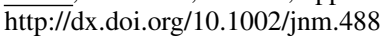

[3] S. Wang, F. L. Teixeira, R. Lee, and J.-F. Lee, "Optimization of subgridding schemes for FDTD," IEEE Microwave and Wireless Components Letters, vol. 12, no. 6, pp. 223-225, June 2002.

[4] K. Xiao, D. J. Pommerenke, and J. L. Drewniak, "A three-dimensional fdtd subgridding method with separate spatial and temporal subgridding interfaces," in Electromagnetic Compatibility, 2005. EMC 2005. 2005 International Symposium on, vol. 2. IEEE, 2005, pp. 578-583.

[5] J. Ritter and F. Arndt, "A generalized 3d subgrid technique for the finitedifference time domain method," in Microwave Symposium Digest, 1997., IEEE MTT-S International, vol. 3, June 1997, pp. 1563-1566 vol.3.

[6] S. Dey and R. Mittra, "A locally conformal finite-difference timedomain (FDTD) algorithm for modeling three-dimensional perfectly conducting objects," Microwave and Guided Wave Letters, IEEE, vol. 7, no. 9, pp. 273-275, September 1997. [Online]. Available: http://ieeexplore.ieee.org/stamp/stamp.jsp?tp=\&arnumber $=622536$

[7] M. R. Cabello, L. D. Angulo, J. Alvarez, A. R. Bretones, G. G. Gutierrez, and S. G. Garcia, "A new efficient and stable 3D conformal FDTD," IEEE Microwave and Wireless Components Letters, vol. 26, no. 8, pp. 553-555, Aug 2016.

[8] J. Beggs, R. Luebbers, K. Yee, and K. S. Kunz, "Finite-difference timedomain implementation of surface impedance boundary conditions," Antennas and Propagation, IEEE Transactions on, vol. 40, no. 1, pp. 49-56, Jan 1992. 
[9] M. Sarto, "A new model for the FDTD analysis of the shielding performances of thin composite structures," Electromagnetic Compatibility, IEEE Transactions on, vol. 41, no. 4, pp. 298 -306, Nov. 1999.

[10] M. R. Cabello, L. D. Angulo, J. Alvarez, I. Flintoft, S. Bourke, J. Dawson, R. G. Martin, and S. G. Garcia, "A hybrid crank-nicolson fdtd subgridding boundary condition for lossy thin-layer modeling," IEEE Transactions on Microwave Theory and Techniques, vol. 65, no. 5, pp. 1397 -1406, May 2017.

[11] M. R. Cabello, S. Fernández, M. Pous, E. Pascual-Gil, L. D. Angulo, P. López, P. J. Riu, G. G. Gutierrez, D. Mateos, D. Poyatos, M. Fernandez, J. Alvarez, M. F. Pantoja, M. Añón, F. Silva, A. R. Bretones, R. Trallero, L. Nuño, D. Escot, R. G. Martin, and S. G. Garcia, "Siva UAV: A case study for the EMC analysis of composite air vehicles," IEEE Transactions on Electromagnetic Compatibility, vol. PP, no. 99, pp. 1-11, 2017.

[12] C. Holloway, M. Sarto, and M. Johansson, "Analyzing carbon-fiber composite materials with equivalent-layer models," Electromagnetic Compatibility, IEEE Transactions on, vol. 47, no. 4, pp. 833-844, Nov 2005.

[13] M. S. Sarto and A. Tamburrano, "Innovative test method for the shielding effectiveness measurement of conductive thin films in a wide frequency range," IEEE Transactions on Electromagnetic Compatibility, vol. 48, no. 2, pp. 331-341, 2006.

[14] L. D. Angulo, M. R. Cabello, J. Alvarez, A. R. Bretones, and S. G. García, "From microscopic to macroscopic description of composite thin panels: A road map for their simulation in time domain," IEEE Transactions on Microwave Theory and Techniques, 2018.

[15] B. Gustavsen and A. Semlyen, "Rational approximation of frequency domain responses by vector fitting," Power Delivery, IEEE Transactions on, vol. 14, no. 3, pp. 1052 -1061, jul 1999.

[16] K. S. Oh and J. Schutt-Aine, "An efficient implementation of surface impedance boundary conditions for the finite-difference time-domain method," Antennas and Propagation, IEEE Transactions on, vol. 43, no. 7, pp. 660-666, Jul 1995.

[17] M. Feliziani, F. Maradei, and G. Tribellini, "Field analysis of penetrable conductive shields by the finite-difference time-domain method with impedance network boundary conditions (inbcs)," IEEE transactions on electromagnetic compatibility, vol. 41, no. 4, pp. 307-319, 1999.

[18] G. Kobidze, "Implementation of collocated surface impedance boundary conditions in FDTD," Antennas and Propagation, IEEE Transactions on, vol. 58 , no. 7, pp. 2394-2403, July 2010.

[19] V. Nayyeri, M. Soleimani, and O. Ramahi, "Modeling graphene in the finite-difference time-domain method using a surface boundary condition," Antennas and Propagation, IEEE Transactions on, vol. 61, no. 8, pp. 4176-4182, Aug 2013.

[20] A. Van Londersele, D. De Zutter, and D. V. Ginste, "An in-depth stability analysis of nonuniform fdtd combined with novel local implicitization techniques," Journal of Computational Physics, vol. 342, pp. 177-193, 2017.

[21] F.-M. Tesche, "Electromagnetic field shielding of a spherical shell-revisited," Forum for Electromagnetic Research Methods and Application Technologies (FERMAT), 2008. [Online]. Available: http://www.e-fermat.org/files/articles/1536cfedc80f6b.pdf

[22] M. Ansarizadeh, "Calculation of ohe shielding effectiveness of carbonfiber composite structures," Ph.D. dissertation, The Department of Electrical and Computer Engineering, Montreal, Quebec, Canada, September 2013.

[23] S. Dey and R. Mittra, "A conformal finite-difference time-domain technique for modeling cylindrical dielectric resonators," IEEE Transactions on Microwave Theory and Techniques, vol. 47, no. 9, pp. 1737-1739, Sep. 1999.

[24] J. F. Dawson, I. D. Flintoft, S. A. Bourke, M. P. Robinson, M. R. Cabello, S. G. Garcia, and J. Alvarez, "Face centered anisotropic surface impedance boundary conditions in fdtd: Improved performance of staircased mesh for shielding problems,", IEEE Transactions on Microwave Theory and Techniques, 2017.

[25] J. Alvarez, L. D. Angulo, A. R. Bretones, and S. G. Garcia, "A comparison of the FDTD and LFDG methods for the estimation of HIRF transfer functions," in Proceedings on Computational ElectroMagnetics And Electromagnetic Compatibility 2013 (CEMEMC13), 2013.

[26] J. Alvarez, L. D. Angulo, A. Bretones, M. Cabello, and S. Garcia, "A leap-frog discontinuous Galerkin time-domain method for HIRF assessment," Electromagnetic Compatibility, IEEE Transactions on, vol. 55, no. 6, pp. 1250-1259, 2013. 